those who presented with status and $37 \%$ of those who had a briefer first seizure. The occurrence of status epilepticus did not appear to have an adverse effect on outcome in the children in this study.

\title{
EEG BACKGROUND ACTIVITY AND ANTICONVULSANT DRUGS
}

The effects of antiepileptic drugs (AED) on EEG background activity in 37 newly treated children with epilepsy were examined at the Departments of Pediatrics, Faculty of Medicine, Toyama Medical and Pharmaceutical University, Toyama City, Japan. Compared to 46 age-matched healthy controls, the EEGs in children with epilepsy, before AED therapy, showed significant slowing. Both idiopathic and symptomatic epilepsies were associated with EEG slowing. Following 3 to 6 months of AED therapy, the EEG slowing was increased in 23 taking carbamazepine for partial seizures and reduced in the 14 treated with valproic acid for generalized seizures. Despite continuous treatment with carbamazepine, after 1 year the background activity had slowly increased in frequency with age. (Konishi $\mathrm{T}$ et al. Effects of antiepileptic drugs on EEG background activity in children with epilepsy: initial phase of therapy. Clin Electroencephalogr April 1995;26:113-119). (Reprints: Tohru Konishi MD, Department of Pediatrics, Faculty of Medicine, Toyama Medical and Pharmaceutical University, 2630 Sugitani, Toyama City, 930-01 Japan).

COMMENT. EEG background activity in children with epilepsy may be slowed because of underlying central nervous system dysfunction related to the epilepsy itself as well as the result of treatment with certain anticonvulsant drugs. Patients with partial epilepsy may be more sensitive to slowing than those with generalized seizures, but drugs such as carbamazepine may exacerbate the tendency to EEG slowing while valproic acid decreases delta activity and is associated with increased EEG frequencies.

\section{SURGERY FOR PARTIAL SEIZURES AND CORTICAL DYSPLASIA}

The role of ictal or continuous epileptogenic discharges (I/CEDs), recorded during intraoperative electrocorticography (ECoG), in the planning of surgical resection for patients with cortical dysplastic lesions (CDyLs) and intractable partial seizures was evaluated at the Montreal Neurological Institute, the Epilepsy Surgery Program, Porto-Alegre, Brazil, and the Chonbuk National Hospital, Chonju, Korea. I/CEDs, consisting of repetitive electrographic seizures, repetitive bursting discharges, or continuous rhythmic spiking, were present in 23 of 34 patients $(67 \%)$ with seizures associated with CDyLs, and in 1 of 40 (2.5\%) whose partial epilepsy was associated with other types of structural leasions. A favorable surgical outcome was obtained in $75 \%$ of patients when the cortical dysplastic tissue showing I/CEDs was completely excised, whereas the outcome was poor if areas containing I/CEDs remained in situ. The authors advocate the removal as much as possible of the cortical dysplastic lesion that is visible and also those areas showing I/CEDs on acute ECoG. (Palmini A, Gambardella A, Andermann F et al. Intrinsic epileptogenicity of human dysplastic cortex as suggested by corticography and surgical results. Ann Neurol April 1995;37:476-487). (Respond: Dr Palmini, Servico de Neurologia, Hospital Sao Lucas-PUCRS, Av Ipiranga 6690, Porto Alegre RS, Brasil CEP 90610-000).

COMMENT. The extent of surgical excision for optimal seizure control in these patients was determined by the intraoperative 
electrocorticographic as well as visual identification of intrinsically epileptogenic dysplastic cortical tissue. Completeness of excision of tissue showing I/CEDs was important for seizure control. The authors found that dysplastic cortex was more epileptogenic than other structural lesions, and patients with cortical dysplasia have a greater tendency to intractable seizures and a higher incidence of status epilepticus than those with other lesions. Previous reports from Montreal have found status epilepticus in 30\% of patients with cortical dysplasia compared to $3 \%$ with epilepsy caused by supratentorial tumors. Life-threatening focal status epilepticus due to occult cortical dysplasia, not revealed by MRI, was successfully treated by surgical excision in 4 patients (Desbiens R, Andermann F et al, 1993; see Progress in Pediatric Neurology II, 1994, p292).

\section{CONGENITAL MALFORMATIONS}

\section{PRENATAL CEREBRAL DYSGENESIS AND CEREBRAL PALSY}

The MRIs of 70 cerebral palsy patients, aged 2 - 16 years, performed between 1989 and 1993 at Kansai Medical University Otokoyama Hospital, were analysed to evaluate the causative roles of pre-, peri-, and postnatal events. The $\mathrm{CP}$ was related to neuronal migration disorders in the embryonal stage in 26 patients. These included pachgyria and polygyria in 8 , schizencephaly in 4 , heterotopia in 4 , agenesis of the corpus callosum in 4 , cerebellar hypoplasia 3 , and disorders of neuronal proliferation, differentiation and histiogenesis in 3 . Vascular disorders were diagnosed in 30, intra-uterine infection in 5 , and birth asphyxia in only 9. The authors conclude that $\mathrm{CP}$ of term infants is frequently the result of prenatal factors, either migration defects or cerebral infarction, and birth asphyxia is a relatively uncommon cause. (Sugimoto $T$ et al. When do brain abnormalities in cerebral palsy occur? An MRI study. Dev Med Child Neurol April 1995;37:285-292). (Respond: Dr Tateo Sugimoto, Department of Paediatrics, Kansai Medical University Otokoyama Hospital, Izumi 19, Yawatashi, Kyoto 614, Japan).

COMMENT. The MRI may be used to identify causes of brain lesions underlying cerebral palsy, and birth asphyxia resulting from obstetrical factors is frequently excluded. In 31 of the 70 infants in this study the CP-related brain abnormalities were clearly developmental and prenatal in origin. In 10 of 30 with vascular lesions the damage had probably occurred in the prenatal period, and in 13 the time of damage was undetermined. The World Federation of Neurology cautions that the term birth asphyxia should be applied only to cases with definite evidence of an asphyxial origin for the neurological disability. Neonatal seizures are the most reliable evidence of intrapartum asphxia. The Apgar score is not the best indicator and most children with CP do not have low Apgar scores at birth. A possible causal relationship of perinatal asphyxia and $\mathrm{CP}$ should require the following: 1) severe newborn acidosis, 2) damage to other organs, and 3) severe neurologic abnormalities in the first 24-72 hours. (see Progress in Pediatric Neurology I, 1991, p333-6).

\section{HIPPOCAMPAL CHANGES IN DOWN'S SYNDROME}

Semiquantitative scales and quantitative computerized image analyses 